\title{
Progress in the applicability of plasma X-ray lasers
}

\author{
T. Kuehl • B. Aurand · V. Bagnoud · B. Ecker · U. Eisenbarth • \\ O. Guilbaud · J. Fils · S. Goette · J. Habib · D. Hochhaus · D. Javorkova • \\ P. Neumayer · S. Kazamias • M. Pittman • D. Ros • J. Seres • \\ Ch. Spielmann • B. Zielbauer $\cdot$ D. Zimmer
}

Published online: 22 January 2010

(C) The Author(s) 2010. This article is published with open access at Springerlink.com

\begin{abstract}
Proposed as satellite-based weapons during the 1980s, X-ray lasing was for a long time only achieved with enormous amounts of pump energy in either nuclear explosions or at kilojoule-class laser installations. During the last few years a tremendous development was achieved, most visible in the realisation of the FEL lasers at DESY and SLAC. As important for a wider applicability is the enormous reduction in pump energy for laser pumped plasma X-ray lasers, which now brings such devices into the range of applications for diagnostics and spectroscopy even in smaller laboratories. Main developments were the transient excitation scheme and the optimized pumping concepts. This paper concentrates on developments at the GSI Helmholtzcenter at Darmstadt aiming towards reliable X-ray laser sources in the range from 50 to several $100 \mathrm{eV}$. The main driving forces for the laser development
\end{abstract}

T. Kuehl $(\varangle)$ · B. Aurand · V. Bagnoud · B. Ecker · U. Eisenbarth · J. Fils ·

S. Goette · D. Hochhaus · D. Javorkova · B. Zielbauer · D. Zimmer

GSI, Darmstadt, Germany

e-mail: T.Kuehl@gsi.de

T. Kuehl · B. Aurand · B. Ecker · D. Zimmer

Johannes Gutenberg-University, Mainz, Germany

O. Guilbaud · J. Habib · S. Kazamias · M. Pittman · D. Ros · D. Zimmer

Université Paris Sud, Orsay, France

J. Seres · Ch. Spielmann

Friedrich Schiller-University, Jena, Germany

B. Ecker · B. Zielbauer

Helmholtz Institute, Jena, Germany

B. Aurand · D. Hochhaus · P. Neumayer

EMMI, Extreme Matter Institute, Darmstadt, Germany

D. Hochhaus · P. Neumayer

Johann-Wofgang von Goethe-University, Frankfurt, Germany 
at GSI are the possible application for the spectroscopy of Li-like ions in the storage ring ESR and the future storage ring NESR at FAIR, and the interest in novel plasma diagnostics.

Keywords X-ray lasers $\cdot$ Laser spectroscopy $\cdot$ Nuclear charge distribution

\section{Introduction}

One of the unique features of the PHELIX laser installation at the GSI Helmholtzcenter at Darmstadt is the combination of the ultra-high intensity laser with the heavy-ion accelerator facility at GSI and its planned extension FAIR [1]. This provides a wealth of opportunities of novel physics applications. One proposal within the SPARC [2] program is the generation of high quality X-ray laser beams for X-ray laser spectroscopy of highly-charged ions in the storage ring ESR and the future NESR. The long range perspective is the study of nuclear properties of radioactive isotopes within the FAIR project.

The pumping requirement for X-ray lasing can be easily derived from basic rate equations. Due to the shortening of the spontaneous emission lifetime the frequency becomes the dominating factor, entering with $v^{4}$. The increase of the required pump power between the microwave maser and the optical laser amounts to 10 orders of magnitude, and explains the time span between the demonstration of masers and lasers. With the additional limitations for the realisation of multiple use of the gain zone by resonators, the minimum pump power for a laser at a wavelength below $30 \mathrm{~nm}$ raises far above $1 \mathrm{GW}$.

The key factor for the realisation of plasma X-ray lasers was therefore the availability and sophisticated application of ultra-short pulse laser sources. By optimisation of the delivery scheme of the pump energy today high repetition rate X-ray lasers at very low pump energy have been achieved. Similarly also X-ray lasers approaching the so-called water window below $5 \mathrm{~nm}$ wavelength can be addressed with well manageable optics despite the need for around $100 \mathrm{~J}$ pump pulses. Another important aspect is beam quality. Here the typical self-seeding plasma X-ray lasers show a deficiency due to their nature as single pass devices. As a remedy, seeding with high-order harmonics created by a separate, dedicated ultra-short pulse laser has been demonstrated recently $[3,4]$.

\section{Plasma creation and population inversion}

The modern approaches are taking full advantage of treating the generation of the active medium for an X-ray laser (XRL) as a dynamic process. It is sufficient to reach the necessary conditions only for a short time. Typical X-ray laser schemes are using transitions in the spectrum of relatively high charge states, e.g. Ni- or Ne-like ionic transitions. Within a plasma plume created by the impact of a high-power laser pulse, these ionic states can be reached at still modest intensities, and will survive for several nanoseconds. To create and maintain the population inversion is, however, complicated by the short lifetime of the highly excited states. To maintain gain and lasing in a quasi steady state situation for even a few nanoseconds this leads to the typical pulse energy requirement of $100 \mathrm{~J}$ and more. 
Fig. 1 Pumping under grazing incidence geometry (GRIP) allows for control of the plasma region where the pump pulse is absorbed. Changing the angle of incidence selects the optimal density region

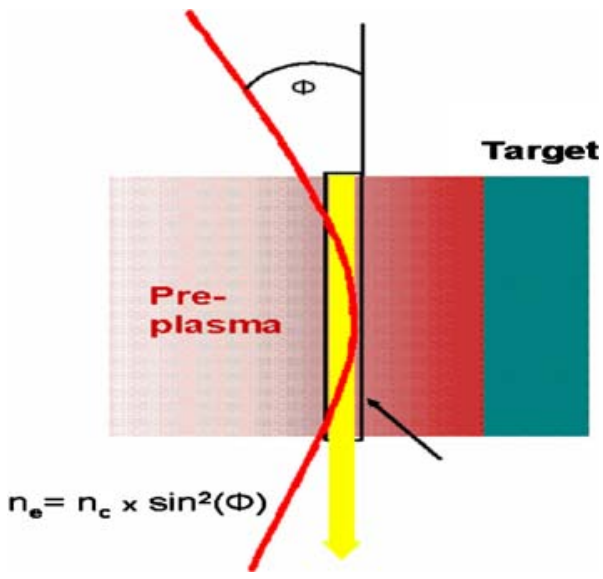

The most important step to ease this requirement drastically was the separation of plasma generation and population inversion. In this way the population inversion is only created and maintained during a few picoseconds. In the original transient collisional excitation (TCE) scheme [5,6], one uses two pulses to irradiate a solid target. The first pulse (pre-pulse) will create a highly ionized plasma, the second pulse (main pulse) will create population inversion in this plasma. The main pulse is shorter in time and as a consequence exhibits a much higher power density than the pre-pulse.

To adapt completely to the inversion population lifetime of less than $5 \mathrm{ps}$, the amplitude front of the short pulse has to be formed to produce a heated region moving with speed-of-light along the preplasma line. This traveling heated region can be associated with the gain region of the XRL. Without this adaptation the short pulse has to have a lifetime of e.g. $30 \mathrm{ps}$ for a $1 \mathrm{~cm}$ focus length. As a result of this technique the required pump pulse energy was reduced to less than $10 \mathrm{~J}$ for lasers down to $13 \mathrm{~nm}$ wavelength. A relatively simple scheme for such an optimised pulse delivery was demonstrated by [7]. Here, the nanosecond pre-pulse was formed into a line focus by a combination of a spherical and a cylindrical lens. For the focusing of the main-pulse a spherical mirror was used under an angle of $45^{\circ}$. In this geometry, with an incoming beam diameter of $7 \mathrm{~cm}$, the astigmatism aberrations create a line focus of about $100 \mu \mathrm{m}$ height and $1 \mathrm{~cm}$ length. Just by the geometrical facts the wavefront hits the target surface in a traveling wave manner with a propagation speed not much above c. In comparison to other set-ups this provided an important simplification. The fact that the main pulse was hitting the target under non-normal incidence was another, even more important feature. This was recognized by [8] in the so-called GRIP scheme, the grazing incidence pumping. Here, as depicted in Fig. 1, the defraction of the pump pulse in the plasma plume is utilised to deposit the pump energy preferentally into the less dense region further away from the target surface, where the optimum conditions for the X-ray lasing can be created. By this a significant reduction of the pump requirement was achieved. Under optimal conditions X-ray lasers in the range below $20 \mathrm{~nm}$ were demonstrated with as little as $100 \mathrm{~mJ}$ pump energy, an improvement of nearly three orders of magnitude compared to the first experiments in the 1990s. 
Fig. 2 Experimental set-up showing the pump laser beam-lines and XRL diagnostics. The insert shows the schematic view of the DGRIP pulse shape. The asymmetric path of the input beam leaves room for a secondary beam for high-harmonics generation

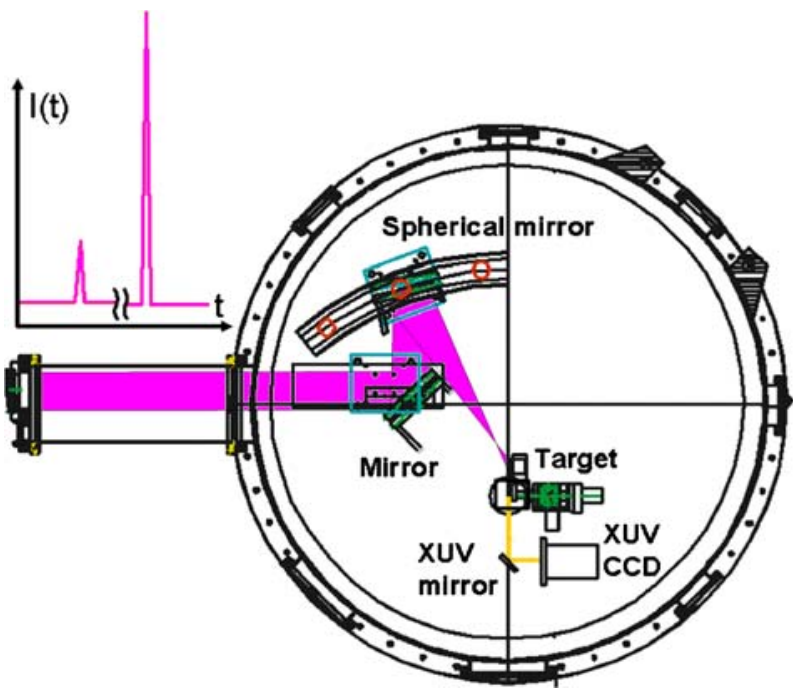

\section{Double pulse grazing incidence pumping}

At GSI an improved pumping scheme for transient collisionally excited soft X-ray lasers was developed. In the usual approach a nanosecond pre-pulse under normal incidence provides the optimal plasma preparation, and a picosecond pulse under non-normal incidence performs the final heating and excitation process. In contrast to this, here both pulses are produced in the front-end of the chirped pulse amplification (CPA) pump laser chain by a small Mach-Zehnder type beamsplitter, and delivered onto the target by the same optics. Using the travelling wave focusing geometry from [7] adjustment of the pump optics is straightforward and stable. The two pulses are focused onto the target with the same spherical mirror under nonnormal geometry, optimized for efficient traveling wave excitation for the mainpulse. A first experiment [8] was performed on Ni-like palladium $(14.7 \mathrm{~nm})$ at less than $500 \mathrm{~mJ}$ total pulse energy on the target. This proves that this configuration is at least as efficient as the standard GRIP scheme, providing much simpler and more reliable operation. The experimental set-up is depicted in Fig. 2: In the first experiments we used a focusing system which produces a line focus with an intrinsic travelling wave speed of $1.2 \mathrm{c}$. The beam from the compressor was deflected by a flat mirror onto a spherical mirror with a focal length of $600 \mathrm{~mm}$ which is positioned off the normal incidence. The line focus on the Pd slab target was $50 \times 5.5 \mathrm{~mm}$ FWHM. Both pulses hit the target at the same grazing incidence angle of $29^{\circ}$, a value determined to be the optimal GRIP configuration in the classical scheme [9, 10]. Thereby the electron density at which the energy is absorbed is matched to the optimum density for X-ray lasing $[11,12]$. The fact that in the double pulse single beam grazinng incidence pumping (DGRIP) scheme [13,14] also the first pulse, which creates the plasma plume, hits the target under the non-normal geometry evidently has no negative effect on the plasma creation. The improved double pulse non-normal incidence pumping scheme provides a simplified and efficient way to produce XRL output photon energy close to $100 \mathrm{eV}$. 

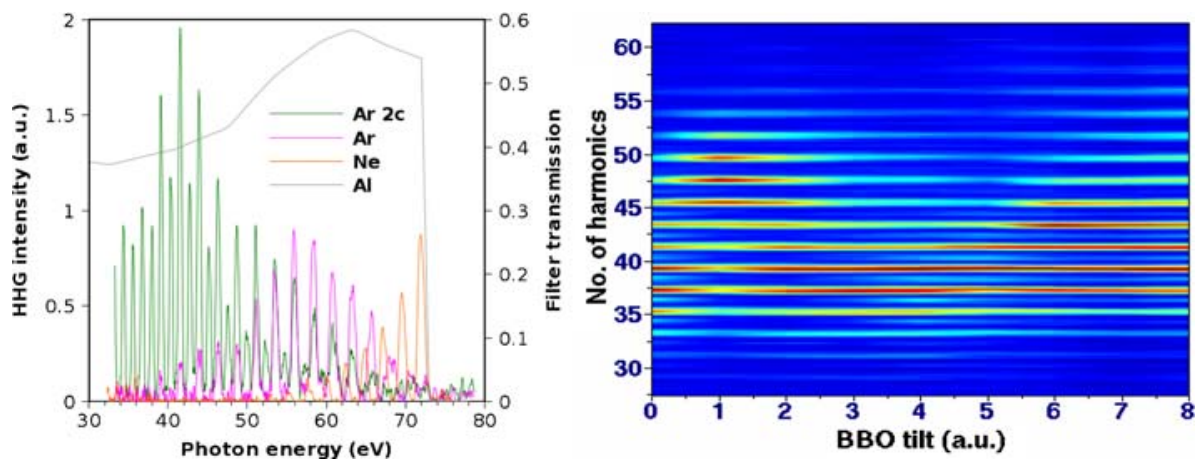

Fig. 3 a Generated harmonics in $\mathrm{Ar}$ with $(\mathrm{Ar} 2 c)$ and without $(\mathrm{Ar}, \mathrm{Ne})$ applying two-color pump. The spectra are cut at the short wavelength side by an Al filter $(A l)$. b The intensity of the even harmonics is sensitive to the tilt angle of the BBO

\section{Seeding of plasma $X$-ray lasers}

Seeding X-ray lasers with high-order harmonic $(\mathrm{HH})$ radiation has been successfully demonstrated recently $[3,4]$, and is under extensive investigation nowadays. Seeding changes the quality of the plasma X-ray laser dramatically, since the injected photons are amplified instead of the spontaneous emission. With high-order harmonic generation a large number of discrete spectral lines at the odd multiples of the driving laser frequency are created in a wide spectrum. Each of these components exhibits excellent spatial and temporal coherence. Unfortunately, most of the harmonic lines do not coincide exactly with the wavelength of potential X-ray laser transitions. Using a Nd:glass laser system such as PHELIX at GSI for pumping an X-ray laser, the wavelength of the fundamental laser beam cannot be tuned. However, harmonic lines can be shifted due to effects induced by the ionization degree and density of the medium [15]. The magnitude of the shift can be controlled e.g. with the gas backing pressure. However, the obtainable shift is not sufficient to cover the whole wavelength range. The number of possible X-ray laser transitions can be increased by using the two-color scheme for $\mathrm{HH}$ generation. Using pulses at the fundamental laser frequency mixed with pulses at the second harmonic, it is possible to generate odd and even harmonics $[16,17]$. We used this scheme to generate strong even and odd harmonics in a wide spectral range in Ar gas suitable for seeding a number of Ni-like X-ray lasers.

The HH source was pumped by the fs-front-end of the PHELIX laser system, operating at $10 \mathrm{~Hz}$ repetition rate. The $10 \mathrm{~mJ}$ pulses have a duration of $300 \mathrm{fs}$, and were focused into the gas jet with a lens with a focal length of $400 \mathrm{~mm}$. The required vacuum condition was ensured by applying a pulsed gas flow. A $0.3 \mathrm{~mm}$ thick BBO crystal was placed into the focused beam. Under optimized conditions the measured second harmonics conversion efficiency was up to $55 \%$. The generated XUV radiation was analyzed by using a flat-field grating $(1,200 \mathrm{l} / \mathrm{mm})$ and a CCD camera in terms of the spectral intensity.

In the experiment we varied the Ar gas pressure for tuning the $\mathrm{HH}$ frequency, and rotated the BBO crystal to adjust the fraction of the second harmonic in the pump (Fig. 3). Using the fundamental beam, only odd harmonics were generated 


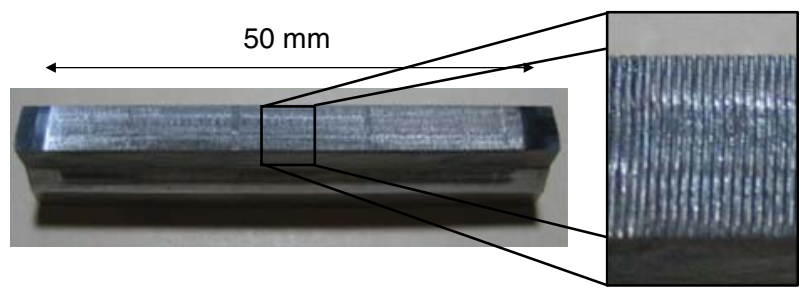

Fig. 4 Molybdenum target after continuous operation of the Ni-like Mo X-ray laser at $18.9 \mathrm{~nm}$ wavelength over several hours. The insert to the right shows the regular groove structure created by the material ablation caused by the pump laser focus line

(Fig. 3a, Ar) and the spectrum extends to photon energies up to $70 \mathrm{eV}$. Converting some part (approx. 40\%) of the infrared laser light into the green and using the twocolor beam for $\mathrm{HH}$ generation, even and odd harmonic lines were observed in the measured spectra (Fig. 3a, Ar 2c). The ratio between the even and odd harmonics decreases at larger photon energies and is strongly affected by the tilting angle of the BBO. Extending the two-color $\mathrm{HH}$ spectrum to higher photon energies needs further systematic investigation and the use of $\mathrm{Ne}$ or He instead of Ar.

The generated $\mathrm{HH}$ lines, in combination with pressure tuning, overlap with the Ni-like $\mathrm{Kr}$ at $37.8 \mathrm{eV}$ (32-order), $\mathrm{Y}$ at $51.7 \mathrm{eV}$ (44-order) and $\mathrm{Zr}$ at $56.3 \mathrm{eV}$ (48-order) X-ray laser transitions [18]. By seeding these X-ray lasers, high resolution spectroscopy on highly charged ions such as Li-like uranium, as can be stored in the ESR storage ring at GSI, are in reach.

\section{High repetition rate capability}

The front-end of PHELIX, with its dedication as injector to the high-energy petawatt amplifier section, does not provide a capability for higher repetition rate operation. The improved DGRIP pumping scheme developed at PHELIX however can significantly also reduce the complications when extended operation at high repetition rates are required. This was demonstrated by applying the new scheme at the LASERIX facility at the Université Paris Sud at Orsay [13, 14, 19]. In this case the function of the Mach-Zehnder unit was extended by the incorporation of a double-pass grating stretcher unit. This allowed for the possibility to choose a longer pre-pulse of several 100 ps to be followed by a sub-picosecond main pulse. Using molybdenum as a target it was found that more than 200 X-ray laser pulses could be generated at a given target position without major degradation of the output energy. By implementation of an automatic target positioner, and a movement of $200 \mu \mathrm{m}$ to the next target position, nearly continuous operation was possible over several hours with a single target of $50 \mathrm{~mm}$ height. The ablation of the target material is visible in a movement of the beam by just a few micrometers. Figure 4 shows the resulting groove pattern in a target after extended use. Each line is caused by the ablation by 200 pump pulses, the grating pattern by the automatic movement of the target. This will also be useful to reach even higher repetition rates as proposed in $[20]$. 


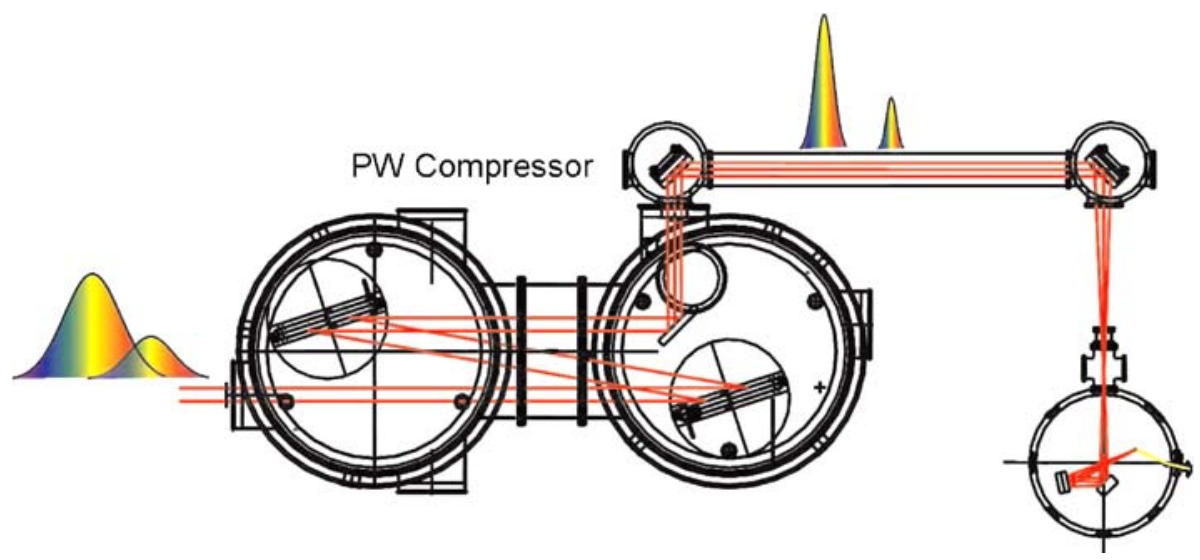

Fig. 5 Beam pass of the high energy pumping arrangement. The double-pulse structure before and after compression is illustrated by the inserts above

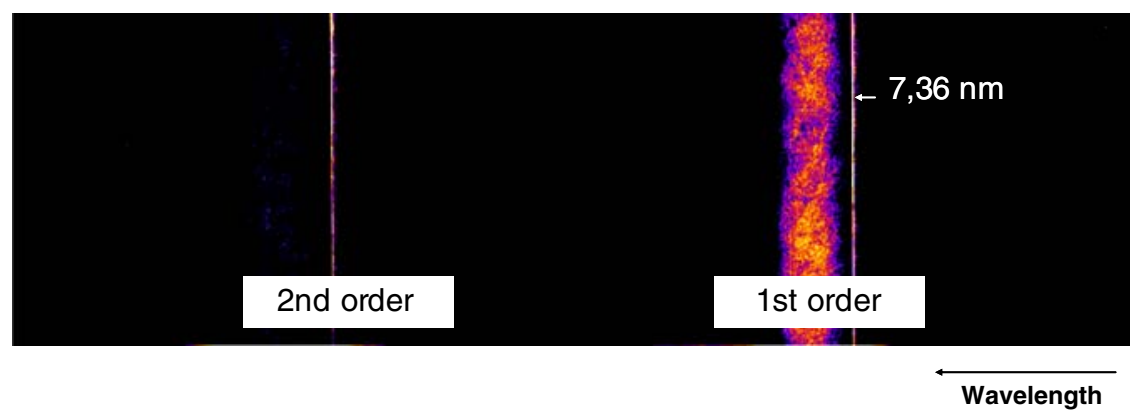

Fig. 6 Spectrum observed for Ni-like samarium pumped with the PHELIX laser

\section{Towards higher energies}

Numerous applications, including imaging of biological specimen and diagnostics of dense plasmas, require coherent X-ray sources exceeding $100 \mathrm{eV}$ photon energy. A main obstacle for such laser sources was the high pump laser energy required for the ionization and the excitation of the lasing transitions. The necessary application of two pulses of different duration and at different angles of incidence was a nontrivial complication for the delivery of high-energy pulses that require the use of large beams. A first experiment using compressed pulses in the range around $50 \mathrm{ps}$ and at energies between 50 and $100 \mathrm{~J}$ at the PHELIX laser therefore incorporated the simplified double pulse scheme demonstrated at the PHELIX frontend, but now at a beam diameter of more than $20 \mathrm{~cm}$. In the first round of experiments [21], a Mach-Zehnder type set-up creates two pulses of identical duration in the front-end of the laser, where the beam diameter is below $5 \mathrm{~mm}$. This double pulse was amplified by the full PHELIX amplifier chain, and recompressed to produce two pulses of 50 to 100 ps duration and with a delay of approximately 1 ns. In this fashion, a line focus for both pulses was produced by a single beam focussing unit consisting of a 
$300 \mathrm{~mm}$ diameter $90^{\circ}$ off-axis parabola and a $200 \mathrm{~mm}$ diameter spherical mirror. The schematics of this set-up is shown in Fig. 5.

A fast photodiode and alternatively a streak camera were used to determine the spacing of the pulses and the relative size of possible pre-pulses. The quality and orientation of the line focus was checked with a microscope viewing the imprint on a glass target at the position of the samarium target. The samarium targets were fabricated at the GSI target laboratory from high purity samarium samples. The X-ray laser output was analyzed by a flat-field grating (1,200 lines per $\mathrm{mm})$ and an XUV CCD-camera. Figure 6 shows a spectrogram of the Ni-like samarium lasing line $7.3 \mathrm{~nm}$, registered at the first and second order of the grating.

In a new series of experiments [22, 23] the Mach-Zehnder set-up was improved by adding a small double pass grating pulse-compressor in one of the arms, similar to the experiments at LASERIX. With this improved set-up the lasing threshold for the Ni-like Sm X-ray laser was reduced to less than 40 J [24]. The results demonstrate the feasibility of applying the simplified double pulse scheme for the pumping of higher energy X-ray lasers. This opens the way to a wider application of such X-ray sources for dedicated X-ray diagnostics of e.g. ion produced plasmas, as well as a future routine operation at facilities like LASERIX [25].

\section{Summary}

The present status of plasma X-ray lasers shows a significant improvement from the situation found just a little time ago. These lasers have become a reliable source of high quality soft X-ray beams, which are seemingly well applicable for different uses. Developments at PHELIX successfully lead to a very reliable set-up, which reaches the needed quality for applications together with accelerator based heavyion experiments. In particular the realization of the sophisticated DGRIP pumping scheme, by the incorporation of a Mach-Zehnder beamsplitter in the very frontend of the laser chain, has served well for the reliable operation and low pump requirements. This opens the way towards a straightforward set-up for seeding with harmonics. The application of this scheme at $10 \mathrm{~Hz}$ repetition rate was demonstrated at LASERIX. Application of the scheme was also demonstrated with high power laser pumping with pulse energies of over $100 \mathrm{~J}$, leading to a Ni-like Sm system with a lasing threshold below $40 \mathrm{~J}$ on target. This could pave the way for higher repetition rate systems even at these shorter wavelength lasers.

Acknowledgements We would like to thank the laser expert teams at PHELIX and LASERIX for their contributions and help. Essential high-energy/high-power laser development relevant for the progress was benefiting from the "Otter" JRA achievements within Laserlab Europe. Part of the work (Ch. Spielmann, J. Seres) was funded by the BMBF program "Hadronen und Kerne". Experiments of the Paris Sud team using high energy pulses at the full PHELIX system were supported by the Laserlab Europe Access program. Daniel Zimmer is thankful for a "Bourse d'Eiffel" grant from Egide.

Open Access This article is distributed under the terms of the Creative Commons Attribution Noncommercial License which permits any noncommercial use, distribution, and reproduction in any medium, provided the original author(s) and source are credited. 


\section{References}

1. Bagnoud, V. et al.: FAIR Conceptional Design Report, GSI. Appl. Phys. B (2009). doi: 10.1007/S00340-009-3855-7

2. Stoehlker, T., et al.: Nucl. Instrum. Methods, B 205, 156 (2003)

3. Zeitoun, Ph., Faivre, G., Sebban, S., et al.: Nature 431, 426 (2004)

4. Wang, Y., Granados, E., Rocca, J.J., et al.: Nature Photonics (February 2008)

5. Nickles, P.V., Shlyaptsev, V.N., Kalachnikov, M.P. et al.: Phys. Rev. Lett. 7, 2748 (1997)

6. Dunn, J., Osterheld, A.L., Shepherd, R. et al.: Phys. Rev. Lett. 80, 2825 (1998)

7. Neumayer, P., Seelig, W., Cassou, K., et al.: Appl. Phys., B 78, 957 (2004)

8. Zimmer, D., Zielbauer, B., Bagnoud, V., Eisenbarth, U., Javorkova, D., Kuehl, T.: Opt. Express 16, 10398 (2008)

9. Keenan, R., Dunn, J., Patel, P.K., et al.: Phys. Rev. Lett. 94, 103901 (2005)

10. Rocca, J.J., Wang, Y., Larotonda, M.A., et al.: Opt. Lett. 30, 2581 (2005)

11. Cassou, K., Kazamias, S., Ros, D., et al.: Opt. Lett. 32, 139 (2007)

12. Kühl, T., Ursescu, D., Bagnoud, V., et al.: Laser Part. Beams 25, 93 (2007)

13. Zimmer, D., et al.: Proc. SPIE 7451, 745108 (2009)

14. Zimmer, D., et al.: Opt. Lett., Doc. ID 117422 (2010)

15. Zair, A., et al.: Phys. Rev. Lett. 100, 143902 (2008)

16. Liu, P., et al.: Phys. Rev., A 79, 063823 (2009)

17. Kim, I.J., et al.: Appl. Phys., B 78, 859 (2004)

18. Seres, J., et al.: Proceedings CLEO (2009)

19. Zielbauer, B., et al.: Appl. Phys., B (2009). doi:10.1007/s00340-009-3877-1

20. Tuemmler, J., et al.: Springer Proc. Phys. 130, 43 (2009)

21. Kuehl, T., et al.: Springer Proc. Phys. 130, 53 (2009)

22. Kuehl, T., et al.: Proc. SPIE 7451, 74510M (2009)

23. Zimmer, D., et al.: Springer Proc. Phys. 130, 91 (2009)

24. Zimmer D., et al.: Proceedings of the Ultrafast Optics Conference (2009)

25. Kazamias, S., Cassou, K., Ros, D., et al.: J. Phys., IV 138, 13 (2006) 